\title{
Der Gemeindebegriff in der Schweiz und die Bedeutung der verschiedenen Gemeinden, dargestellt am Beispiel der Gemeinde Altstätten
}

\author{
Ernst Gächter
}

\section{Einführung}

Oft verwenden wir das Wort Gemeinde und sind uns gar nicht bewußt, wie vieldeutig dieser Begriff sein kann. Meist meinen wir damit eine Politische Gemeinde und vergessen, daß es daneben noch eine ganze Reihe weiterer Gemeinden geben kann, die sich in bezug auf Gebiet und Mitglieder nur bedingt decken. Oft kennen sich nicht einmal Ortsansässige in der Fülle der Gemeinden aus. Deshalb ist es begrieiflich, daß Außenstehende oder gar Ausländer sich nicht zurechtfinden in den schweizerischen Verhältnissen, die von Kanton zu Kanton noch stark variieren.

Der vorliegende Artikel versucht, Bedeutung und Aufgaben der verschiedenen Gemeinden an einem konkreten Beispiel klarzulegen und damit etwas Licht in die Stellung der Schweizer Gemeinde zu bringen. Nach Bader bedeutet gemein, gmeind unverteiltes Land (Allmende). Der Begriff "Gemeinde» hätte somit ursprünglich mehr wirtschaftliche als politische Bedeutung gehabt.

Für die Namengebung in den Kartenskizzen wurde die Schreibweise der Landeskarte 1:25000 übernommen, im Text dagegen wurde für Gemeinden und Rhoden die ortsübliche Schreibweise verwendet, so daß kleinere Abweichungen vorkommen (Warmesberg-Warmisberg z. B.).

Ursprung der Gemeinden und gesetzliche Grundlagen im Kanton St. Gallen

Nach der heute rechtskräftigen Kantonsverfassung von 1890 und dem Organisationsgesetz von 1947 gibt es im Kanton St. Gallen folgende öffentlichrechtlich anerkannte Gemeindetypen und Korporationen:

\section{Politische Gemeinden}

Ortsbürgergemeinden und ortsbürgerliche Korporationen (Unterabteilungen von Ortsgemeinden oder mehrere Ortsgemeinden umfassende Organisationen)

Primar- und Sekundarschulgemeinden

Kirchgemeinden und kirchliche Korporationen (Filial- und Kapellgenossenschaften) katholischer und evangelischer Konfession örtliche Korporationen (Körperschaften mit öffentlichen Aufgaben, zum Beispiel Wasserverşorgung, Beleuchtung).

Der Begriff Gemeinde im heutigen Sinne wird erstmals in den Kantonsverfassungen der Helvetik und Mediation erwähnt. Die neugeschaffenen Gemeinden übernahmen die Aufgaben von bestehenden Organisationen beziehungsweise wurden als solche staatlich anerkannt.

Die Orts- oder Bürgergemeinde ist nach Schöbi aus den alemannischen Sippengemeinschaften und den mittelalterlichen Wirtschaftsgemeinden hervorgegangen. Sie war vor der Französischen Revolution für das Wohl aller Bewohner des Gemeindebannes verantwortlich. Die freie Nutzung ihres großen Grundbesitzers stand anfänglich allen Ortsansässigen zu. Als die Niedergelassenen aber immer mehr zunahmen und das Armenwesen von der Kirche an die Ortsgemeinden übertragen wurde, mußte die Nutzungsberechtigung auf die Ortsbürger eingeschränkt werden.

Die Helvetik schuf mit dem Gesetz über die Bürgerrechte von 13. Hornung 1799 die Politische Gemeinde, der dann der Großteil der Funktiohen der Ortsgemeinde übertragen wurde. Ortsgemeinde und Politische Gemeinde bestanden fortan nebeneinander; in der Verfassung von 1831 wurde die Trennung endgültig durchgeführt und der Gemeindedualismus im Gesetz verankert. In der Folge übertrugen die meisten Ortsgemeinden die Armenunterstützung der Politischen Gemeinde, unter Bezahlung einer einmaligen Abkurung. Organisation und Aufgabe der heutigen Orts- und Politischen Gemeinde sind im Organisationsgesetz von 1947 niedergelegt. Für die Ortsgemeinden besteht zusätzlich noch die Verordnung über die Bewirtschaftung und Nutzung der Ortsgemeindegüter von 1948.

Das Schulwesen wurde vor 1861 von privaten oder konfessionellen Organisationen betreut. Der Unterhalt von Räumlichkeiten und Lehrkräften wurde durch Bezahlung eines Schulgeldes (oft in Form von Naturalien) bestritten. Die Kantonsverfassung von 1861 übertrug das Erziehungswesen der staatlichen Aufsicht, allerdings unter Beibehaltung der bestehenden konfessionellen Schulen, die zu eigenen Gemeinden gemacht wurden. Organisation und Aufgabe der Schulgemeinden sind im Erziehungsgesetz von 1952 festgehalten. 


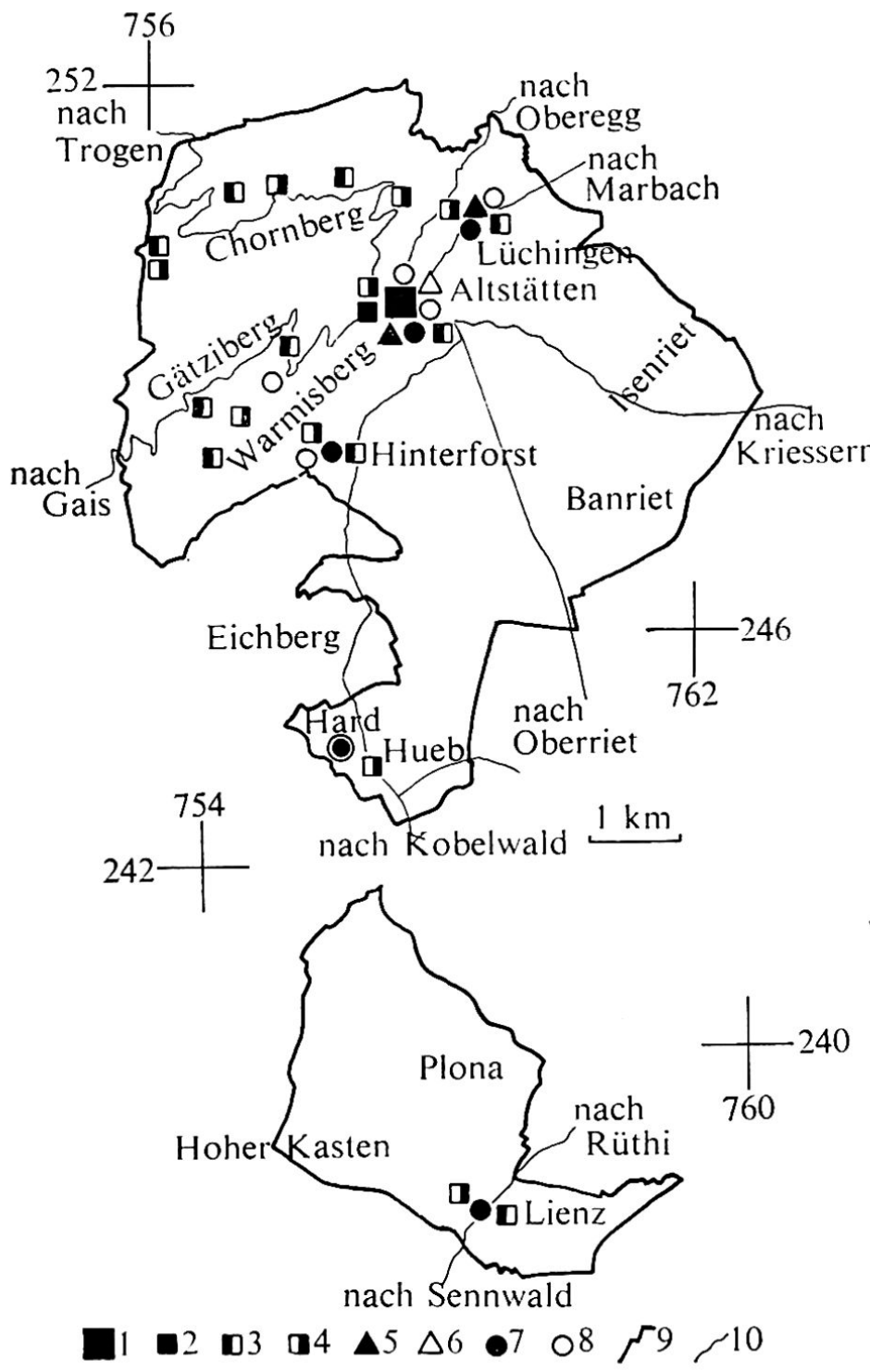

Abbildung 1. Altstätten mit Enklave Lienz: 1 Politische Gemeinde; 2 Ortsgemeinde; 3 Trattrhoden; 4 Holzrhoden; 5 katholische Kirchgemeinden; 6 evangelische Kirchgemeinden; 7 katholische Schulgemeinden; 8 evangelische Schulgemeinden; 9 Grenze der Politischen Gemeinde: 10 Straßen

Die Kirchen gehen meist auf Stiftungen von Adligen zurück. Der Unterhalt der Kirche und der Geistlichen wurde aus Pfründen (Stiftungen von Adligen und Bürgern) und Naturalgaben der Kirchgenossen bestritten. Die Kantonsverfassung von 1814 betont die Autonomie der Konfessionen. Die Verfassung von 1890 bestimmt in Artikel 24, daß die religiösen und kirchlichen Angelegenheiten in den Zuständigkeitsbereich der kirchlichen Behörden fallen, ihre Organisation aber der Sanktion des Großen Rates untersteht. Im Gesetz über die Besorgung der Angelegenheiten des katholischen und des evangelischen Konfessionsteiles von 1923 wird festgelegt, daß die evangelischen kirchlichen Behörden für die Besorgung aller kirchlichen und religiösen Angelegenheiten, die katholischen Behörden aber nur für die nicht rein kirchlichen Belange verantwortlich seien.
Die rein kirchlich-religiösen Bereiche werden katholischerseits durch den Codex Iuris Canonici (Kir. chenrecht) geregelt. Die Evangelische Kirche des Kantons St. Gallen hat sich 1922/23 das heute gül. tige Grundgesetz und die Kirchenordnung gegeben Das gültige administrative Grundgesetz für den ka. tholischen Konfessionsteil datiert von 1939. Im An. hang an die betreffenden Erlasse ist die Aufteilung des Kantons in katholische und evangelische Kirch. gemeinden festgelegt und von den kantonalen $\mathrm{Be}$. hörden bestätigt worden.

\section{Bestand und Zugehörigkeit}

Auf dem Gebiet der Politischen Gemeinde Altstätten bestehen heute die folgenden öffentlich. rechtlich anerkannten Gemeinden und Korporationen (Abb. 1):

Politische Gemeinde Altstätten (mit Enklave Lienz) Ortsgemeinde Altstätten mit zehn Trattrhoden (Stadt und Vorstadt, Lüchingen, Vorderkornberg: Vierhöfe, Loch, Gätziberg-Vorderer Teil, Gätziberg-Lauterbach, Warmesberg-Wasentratt, Hinterforst und Lienz) und neun Holzrhoden (Stadt und Vorstadt, Lüchingen, Kornberg, Loch, Gätziberg. Warmesberg, Hinterforst, Hub-Kobelwies, Lienz und die Reisrhode Wieswanne)

Zehn Schulgemeinden (Katholisch Altstätten, Evangelisch Altstätten, Katholisch Lüchingen, Evangelisch Lüchingen, Katholisch Hinterforst, Evangelisch Hinterforst, Evangelisch Gätziberg-Warmesberg, Katholisch Lienz, die Evangelische Sekundarschulgemeinde Oberrheintal und die Paritätische Schulgemeinde Hub-Hard)

Drei Kirchgemeinden (Katholisch Altstätten, Evangelisch Altstätten und Katholisch Lüchingen)

Im weiteren besteht noch je eine katholische und evangelische Waisenguts- und Fondgemeinde mit Kinderheim und Landwirtschaftsbetrieb in Altstätten.

Einzelne Teil der Politischen Gemeinde gehören in Schule und Kirche zu anderen, außerhalb des Gebietes liegenden Gemeinden, Katholisch Hub-Hard zum Beispiel zur Kirchgemeinde Kobelwald, Evangelisch Hub-Hard dagegen zur Kirchgemeinde Eichberg, Katholisch Lienz und Plona gehören zur Kirchgemeinde Rüthi, wobei Lienz eine Kapellgenossenschaft bildet, Evangelisch Lienz gehört zur Kirch- und Schulgemeinde Sennwald, Katholisch Lüchingen bildete bis vor kurzem noch einen Teil der Kirchgemeinde Marbach.

Diese komplizierten Verhältnisse spiegeln sich in der Steuererhebung, die innerhalb der Gemeinde zwölf verschiedene Gruppen umfaßt (Tab. 1). Jeder Steuerpflichtige in Altstätten muß Staats-, Gemeinde- und Schulsteuern und, falls er einer der beiden staatlich anerkannten Konfessionen angehört, 
Tabelle 1. Politische Gemeinde Altstätten: Bezugsgruppen und Steueransätze für 1966 (in Prozenten der einfachen Staatsteuer), Steuerpflichtige nach Bezugsgruppen

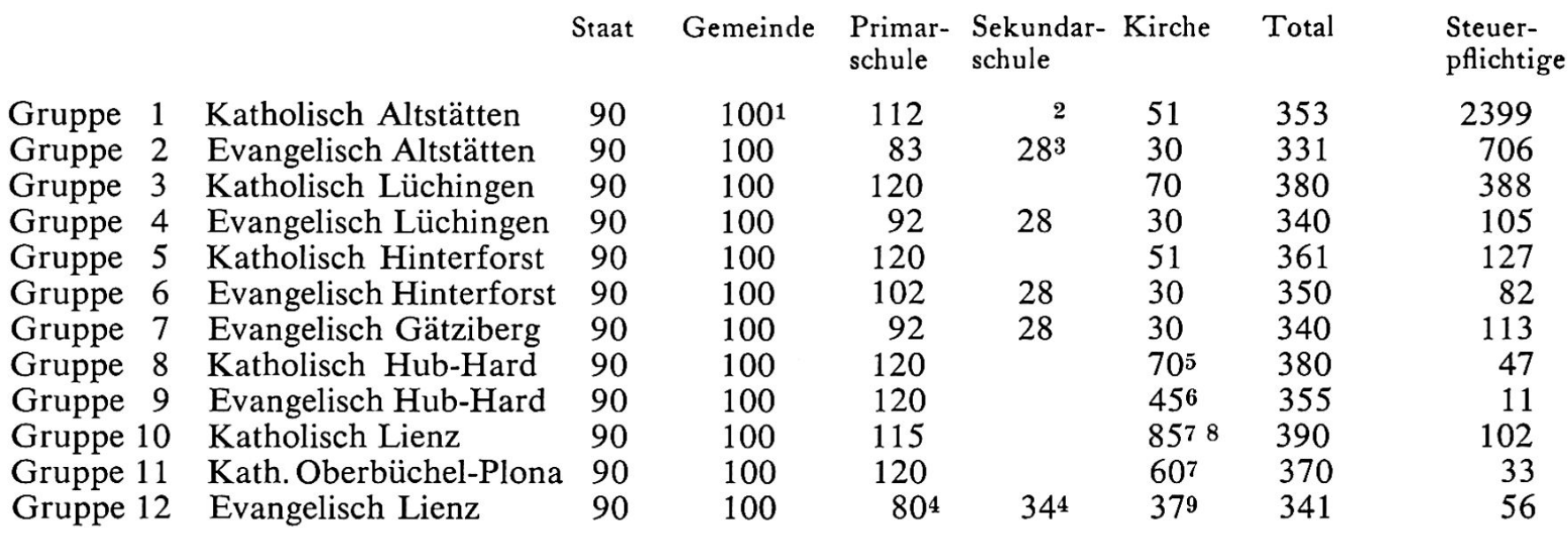

$170 \%$ allgemeiner Gemeindehaushalt, 30\% Fürsorgehaushalt; 2 Katholische Sekundarschulsteuer inbegriffen in Primarschulsteuer; ${ }^{3}$ Evangelische Sekundarschulgemeinde Oberrheintal; 4 Evangelische Primarbzw. Sekundarschulgemeinde Sennwald; 5 Katholische Kirchgemeinde Kobelwald; 6 Evangelische Kirchgemeinde Eichberg; 7 Katholische Kirchgemeinde Rüthi; 8 einschließlich 25\% Kapellsteuer Lienz; ${ }^{9}$ Evangelische Kirchgemeinde Sennwald

auch die betreffende Kirchensteuer zahlen. Ein Konfessionsloser kann die Schulgemeinde unter den gemäß seinem Wohnsitz möglichen Schulgemeinden frei wählen. Er bezahlt dieser die Schulsteuer und muß seine Kinder auch dort in den Unterricht schicken.

1960 betrug die Einwohnerzahl der Politischen Gemeinde Altstätten 8751 Personen. Davon waren 3617 Ortsbürger, 4628 übrige Schweizer Bürger und 506 Ausländer. Altstätten selbst zählte 5696 Einwohner, Lüchingen 795, Hinterforst 263, Lienz 247, 750 verteilten sich auf das übrige Gemeindegebiet (Kornberg, Gätziberg, Warmesberg, HubHard, Plona usw.). 6622 Einwohner gehörten der katholischen und 2107 der evangelischen Konfession an, 22 bekannten sich zu einer anderen Konfession oder waren konfessionslos.

\section{Die Politische Gemeinde Altstätten}

Die Politische Gemeinde erfüllt alle kommunalen öffentlichen Aufgaben, soweit sie darin nicht durch kantonale oder eidgenössische Gesetze eingeschränkt ist oder die Aufgaben durch das Gesetz anderen Gemeinden zugewiesen sind. Es seien genannt: Polizei, Feuerwehr, Wasser-, Elektrizitätsund Gasversorgung, Abwasser, Grundbuchamt, Zivilstandsamt, Armenwesen usw. Daneben sind ihr von Kanton und Bund gewisse Aufgaben übertragen (AHV, Militär u. a.).

Altstätten entwickelt sich in jüngster Zeit stark, nachdem die Bevölkerungszahl lange konstant bis rückläufig gewesen war und Industrie und Handel sich während Jahrzehnten nur langsam ausdehnten. Deshalb steht die Gemeinde vor großen Auslagen für den Ausbau der Infrastruktur. Es seien erwähnt der Bau einer Abwasserkanalisation, einer Kläranlage und die Erweiterung des Straßennetzes.

Die Politische Gemeinde umfaßt alle am Ort niedergelassenen Schweizer Bürger. Ihre Einnahmen bezieht sie vor allem aus den Gemeindesteuern, die auch von den in der Gemeinde wohnhaften Ausländern bezahlt werden müssen. Die Politische Gemeinde zieht zusammen mit den Staats- und Gemeindesteuern auch die Steuern für die verschiedenen Schul- und Kirchgemeinden ein.

\section{Die Ortsgemeinde Altstätten}

Die Ortsgemeinde Altstätten ist wegen ihrer Größe in neunzehn Rhoden (Verwaltungskorporationen) aufgegliedert. Die ausgedehnten Kulturland- und Weideflächen waren schon seit unbekannter Zeit aufgeteilt und einzelnen Hofgruppen zur Nutzung zugewiesen worden; 1741 wurde auch der große Waldbesitz geteilt, so daß heute noch jeder Ortsbürger in der Ortsgemeinde selbst und je nach seinem Wohnort innerhalb der Gemeinde in einer Tratt- und Holzrhode nutzungsberechtigt ist. In erster Linie gilt das Familiensystem für die Nutzungsberechtigung, bei ledigen Ortsbürgern wird eine Kombination von Kopf- und Haushaltungssystem (zurückgelegtes 25. Altersjahr und Führung eines eigenen Haushaltes) angewendet. 


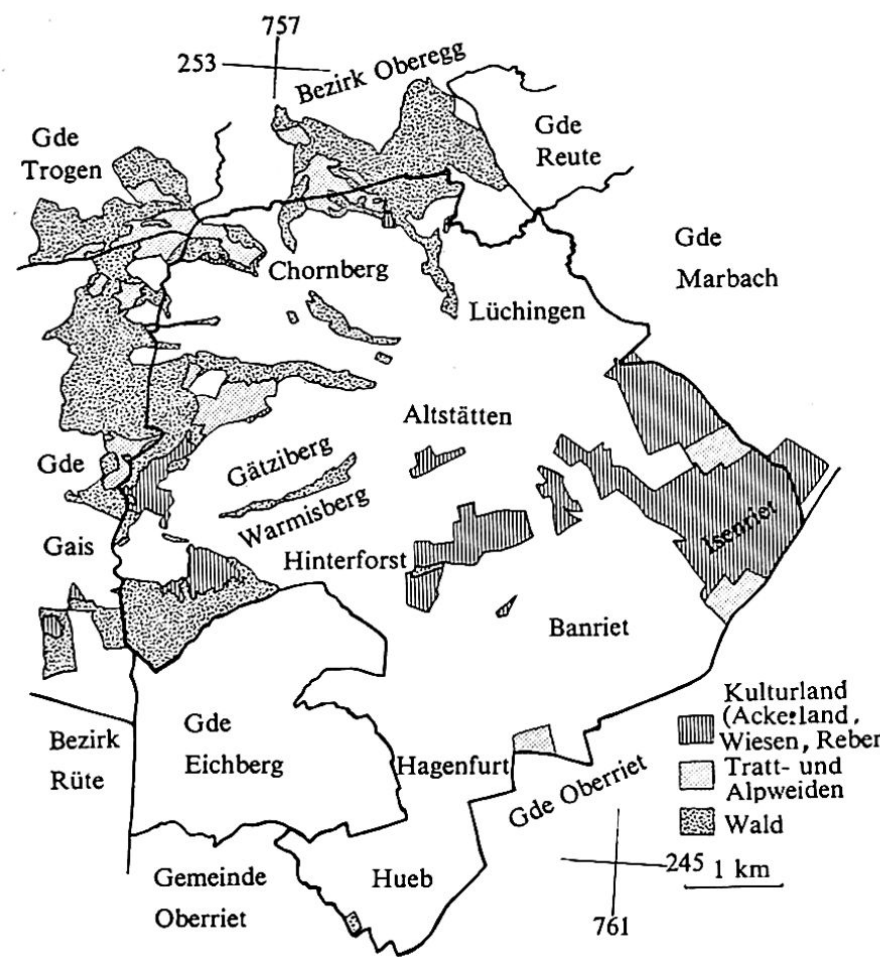

Abbildung 2. Ortsgemeinde Altstätten und Rhoden: Nutzung des Grundbesitzes

Bestand und Organisation von Ortsgemeinde und Rhoden sind (neben den kantonalen Gesetzen) in Vereinbarung und Reglement über die Gemeindegüterverwaltung der Ortsgemeinde Altstätten von 1950 festgehalten. Der Ortsgemeinde stehen die folgenden Aufgaben und Pflichten zu: Bewirtschaftung der sich im Besitz der Ortsgemeinde befindenden Güter, Aufsicht über die Rhoden (Verwaltung, Bewirtschaftung der Güter, Vermittlung bei Streitigkeiten über die Rhodszugehörigkeit usw.), Führung des Bürgerregisters, Behandlung von Einbürgerungsgesuchen und die Unterstützung von gemeinnützigen Werken.

Der Grundbesitz der Ortsgemeinde umfaßt heute 13,7 ha unverteiltes Land (2,5 ha Reben, 5,5 ha Wiesland und 4,7 ha Wald), weitere 1382,7 ha werden durch die einzelnen Rhoden verwaltet. Auf Abbildung 2 können wir zwei Hauptgebiete des Ortsgemeindebodens feststellen: die abgelegensten Teile der Rheinebene und die mit Wald und Alpweiden genutzten obersten Talhänge. Im letzteren Gebiet reicht der Grundbesitz weit über die Grenzen der Politischen Gemeinde hinaus, da diese durch die Appenzeller nach der Schlacht am Stoß 1405 von der Wasserscheide gegen das Rheintal hinunter verlegt wurde und seither so blieb.

Die Ortsgemeinde Altstätten leistet mit der Pflege ihres Rebberges einen bedeutenden Beitrag an die Erhaltung des Rebbaus im Rheintal. Der Wein, der heute noch in einer 300jährigen Trotte von Hand gepreßt wird, ist unter dem Namen Altstätter Forstwein bekannt. Früher wurde er sofort nach der Pressung in Schlägen zu 200 oder 400 Liter verstei- gert. Da jedoch die Qualität des Weines unter deı oft unfachmännischen Lagerung litt, wird heute deı gesamte Ertrag nach der Pressung von zwei Groß. firmen übernommen und gelagert. Der Wein kanr dort dann in Schlägen oder en détail gekauft wer. den.

Die Ortsgemeinde hat bei Einbürgerungen gegen. über der Politischen Gemeinde die Priorität. Es wird nur alle zwei bis drei Jahre jemand ins Bürger. recht aufgenommen, da die Ortsbürgerversammlun§ Gesuchen sehr zurückhaltend zustimmt. 1966 muß. te ein lediger Österreicher 2500 Fr. zahlen und aut die Nutzungsberechtigung verzichten, um aufge. nommen zu werden.

Laut Artikel 148 des Organisationsgesetzes ist dit Ortsgemeinde verpflichtet, Beiträge an gemeinnüt. zige Werke zu leisten. In jüngster Zeit wurden jє 200000 Fr. an die Kläranlage Altstätten und dif zu bauende Mittelschule Rheintal durch die Orts. gemeinde und die Rhoden beschlossen. Auch Stra. ßen- und Brückenbauten werden unterstützt.

Die Verwaltungsrechnung der Ortsgemeinde Alt.

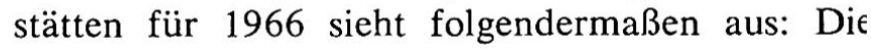
wichtigsten Posten der Einnahmeseite waren:

Bodenverkäufe 40000 Fr., Erlös aus dem Weinbac 24000 Fr., Ertrag der Wertschriften $4000 \mathrm{Fr}$. Pachtzinsen 2100 Fr.; die Ausgaben verteilten sich auf Straßenbauten 40000 Fr., Aufwendungen füı den Rebberg 25000 Fr., Verwaltungskosten $410 \mathrm{C}$ Fr., Steuern 2200 Fr.

Das Vermögen beläuft sich auf 200000 Fr., davon sind 80000 Fr. Ertragswert des Grundeigentums und 120000 Fr. Obligationen, Aktien und Spar. hefte. Im weiteren bestehen noch zwei zweckge. bundene Fonds von zusammen 85000 Fr. (Lehr. lingsfonds und Marolanischer Baufonds zur Ver. schönerung des Stadtbildes). Einkommen und Ver. mögen der Ortsgemeinde sind steuerpflichtig, die Ortsgemeinde selbst muß ihre laufenden Ausgaben aus den Einnahmen decken und darf keine Steuern erheben.

\section{Die Riettrattrhode Stadt und Vorstadt Altstätten}

Als Beispiel einer ortsbürgerlichen Korporation seien die Verhältnisse in der Trattrhode Stadt und Vorstadt dargestellt. Die Rhode zählte 1948 540, 1965 noch 530 nutzungsberechtigte Genossen. Der Grundbesitz umfaßt 211 ha Kulturland, das alles in der Rheinebene liegt. Es wird auf die folgenden Arten genutzt: Hofstattboden, Pflanzgärten, Gemeindegüter, Pachtlose, Turbenrechte und die Trattweide (Abb. 3).

Im Hofstattboden hat sich ein alter Rechtszustand bis in die heutige Zeit erhalten: Der Grund gehört der Rhode, die Gebäude dagegen verschiedenen Rhodsgenossen (meist Bauern), die einen Hofstatt- 


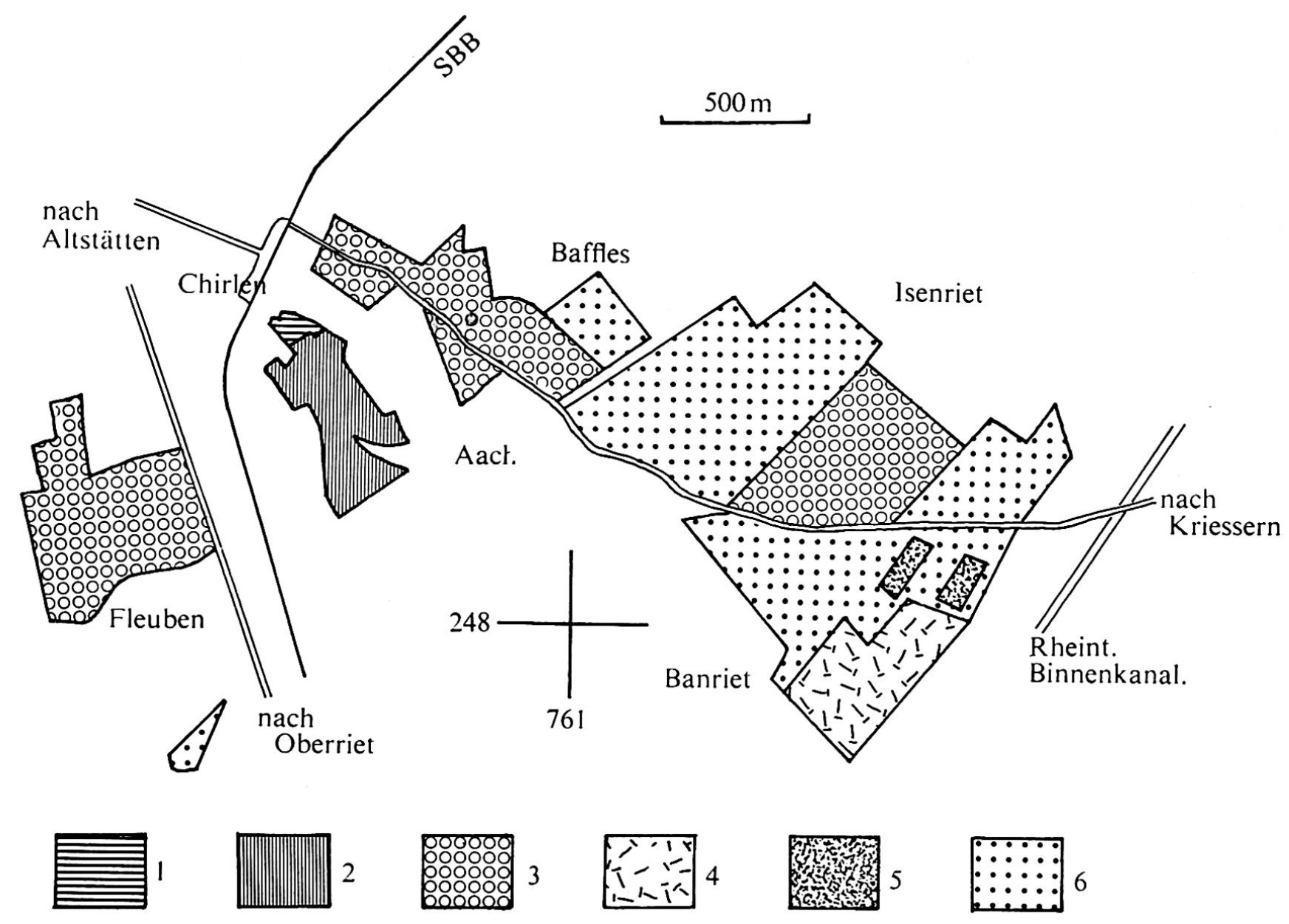

Abbildung 3. Grundbesitz der Riettrattrhode Stadt und Vorstadt Altstätten: 1 Hofstätten; 2 Pflanzgärten; 3 Genossengüter; 4 Trattweide; 5 Torfland; 6 Pachtlose

zins von 4 Rp. je Quadratmeter jährlich zahlen. Seit der Einführung des Schweizerischen Zivilgesetzbuches ist dieser Zustand rechtlich nicht mehr erlaubt (ZGB Art. 675, 779). 1964/65 wurde eine Reihe von Hofstatten aufgelöst, die 28 noch bestehenden im Unterchirlen (Abb. 4) werden demnächst folgen. Die Rhodsgenossen müssen das Land von der Rhode kaufen oder ein dauerndes Baurecht als Dienstbarkeit im Grundbuch eintragen lassen.

In Ortsnähe hat die Rhode 140 Pflanzgärten zu 4 und 8 a ausgeschieden. Der Pachtzins beträgt 4 Fr. je Are. Nach dem Zweiten Weltkrieg bestand eine große Nachfrage nach Gemüsegärten zur Selbstversorgung; heute wird das Areal zum Teil auch als Wiesland genutzt (Abb. 4).

Jeder Rhodsgenosse hat Anrecht auf 32 a Gemeindegut (zu einer jährlichen Auflage von $10 \mathrm{Fr}$.), das er aber selbst bewirtschaften muß. 1948 zählte die Rhode 220 Genossen, die das Gemeindegut nutzten, 1965 noch 160. Die Nutzungsdauer ist unbeschränkt, solange das Land selbst bewirtschaftet wird, es kann auf Witwe oder Nachkommen übertragen werden.

Aus dem Land, das nicht für Gemeindegüter oder andere Sonderzwecke gebraucht wird, wurden Pachtlose von 30 bis 400 a gemacht. Der Pachtzins beläuft sich auf Fr. 2.50 bis Fr. 3.50 je Are. Dem einzelnen Bewirtschafter werden möglichst nebeneinanderliegende Parzellen gegeben. Die Pachtdauer beträgt 10 Jahre, kann aber beliebig verlängert werden, sofern das Land von der Rhode nicht benötigt wird.

Im äußeren Eisenriet besitzt die Rhode noch unmelioriertes, hohes Torfland, das einen Meter abgegraben werden darf. Die Verpachtung erfolgt als sogenanntes Turbenrecht, das heißt die Erlaubnis, eine bestimmte Anzahl Kubikmeter Torf (= Turben im Rheintaler Dialekt) abgraben zu dürfen. Der Pachtzins beträgt Fr. 1.50 je Kubikmeter für Rhodsgenossen und 2 Fr. für Fremde. Das Turbenstechen ist ein aussterbendes Gewerbe, das heute nur noch von alten Leuten ausgeübt wird. Es sind noch etwa 80 Interessenten an Turbenrechten vorhanden, die meist aus den Rheindörfern mit wenig Wald kommen (Krießern vor allem). Der Torf war früher im Rheintal beliebt als Koch- und Heizmaterial, heute wird er auch zu Torfmull verarbeitet.

Die Trattweiden sind eine für das Rheintal typische Form von gemeinschaftlicher Viehweide in der Rheinebene. Sie gehören alle den Ortsgemeinden und sind als letztes Relikt des früheren, unbeschränkten und allgemeinen Weidgangs auf der Allmend zu betrachten. Die Tratt im Eisenriet war früher ein Eigenbetrieb der Rhode mit einem ange- 
stellten Hirten. Heute ist sie mit der Auflage der Weiterführung des Trattbetriebes an einen in der Nähe wohnenden Bauern verpachtet. Die Tratt umfaßt 14 ha schlechten Torfboden und einen Trattstall für 72 Stück Vieh. Es wird hier ausschließlich Jungvieh einige Wochen vor und nach der Hochalp geweidet.

Die Rhodsgenossen, die kein Genossengut bewirtschaften, erhielten 1966 eine Entschädigung von 60 Fr. (1965 50 Fr.). Die Verwaltungsrechnung der Rhode für 1965 sah folgendermaßen aus:

Ausgaben: Entschädigung an Stelle von

Genossengut

Fr. 18500

Unterhaltskosten von Wegen

usw.

Fr. 11800

Steuern

Fr. 8100

Meliorationsperimeter

Fr. 7100

Verwaltungskosten

Fr. 3800

Einnahmen: Zins für Pachtlose

Fr. 32600

Bodenverkäufe (Hofstatt-

boden)

Fr. 14500

Pachtzinsen für

Turbenrechte

Fr. 6600

Kapitalzinsen

Fr. 4700

Pachtzinsen für

Pflanzgärten

Fr. 3300

Auflage für Gemeindegüter

Fr. 2400

Das Vermögen der Rhode beläuft sich auf 1360000 Fr. (1 114000 Fr. Ertragswert des Bodens, 35000 Fr. Trattstall Burst, 115000 Fr. Obligationen, Aktien und Sparhefte).

Zur Bodenpolitik der Rhode ist zu bemerken, daß Land nur gegen Realersatz verkauft wird, trotz groBer Nachfrage nach Bau- und Industrieland. Da viele Bauern auf das Rhodsland angewiesen sind, erfüllt dieses eine wichtige Funktion in der Erhaltung der Landwirtschaft.

\section{Die katholische Schulgemeinde Altstätten}

Weil die Politische Gemeinde Altstätten neben der Stadt noch einige Dörfer und Berggebiete umfaßt und die Schulen bis heute konfessionell getrennt sind, finden wir eine ganze Reihe von Schulgemeinden, die zum Teil sehr klein sind.

Als Beispiel sollen im folgenden die Verhältnisse in der katholischen Schulgemeinde Altstätten geschildert werden. Um 1800 bestanden je eine katholische Unter- und Oberschule in der Stadt und sechs Rhodsschulen im übrigen Gemeindegebiet. Der Schulbesuch war nicht obligatorisch und, außer in der Oberschule, der Unterricht nicht ganzjährig. 1859 wurde die katholische Realschule gegründet.

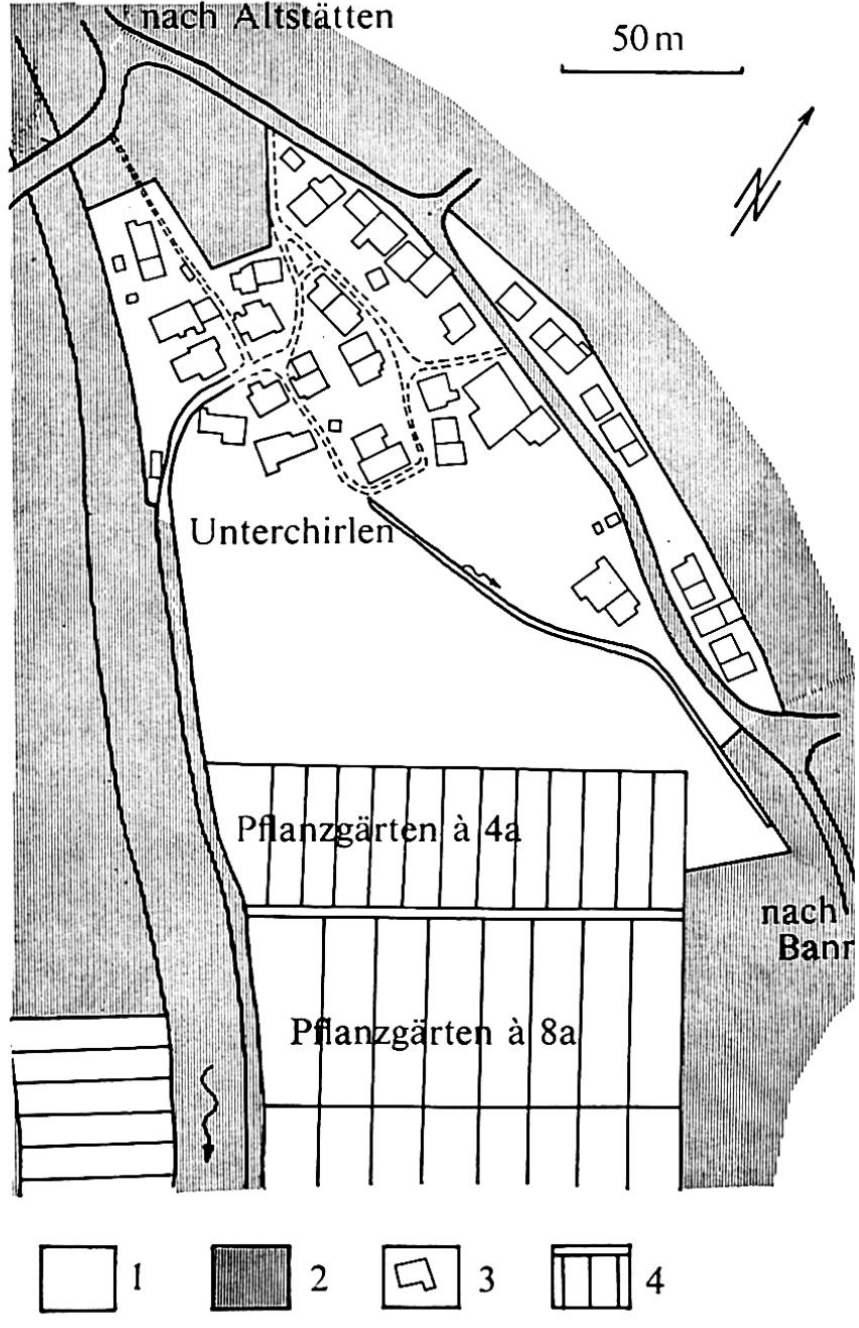

Abbildung 4. Riettrattrhode Stadt und Vorstadt Alt stätten: Nutzungsplan Unterchirlen: 1 Grundbesitz der Rhode 2 andere Grundbesitzer; 3 Hofstätten 4 Pflanzgärten

1864 übertrug die Schulgemeinde die Primar- unc Sekundarschule für Mädchen dem Kloster Maric Hilf in Altstätten, wo sie bis in jüngste Zeit blieb Zwischen 1920 und 1930 wurden die damals selb. ständigen katholischen Bergschulgemeinden Vor. derkornberg, Hinterkornberg-Ruppen und Gätzi. berg inkorporiert. Vor einigen Jahren wurde aut Wunsch der Lehrer die Koedukation eingeführt, nur das Kloster führt noch eine reine Mädchensekun. darschule.

Heute bestehen in der katholischen Schulgemeinde Altstätten zwei Primar- und ein Sekundarschulhaus in Altstätten selbst und je ein Primarschulhaus am Vorderen und Hinteren Kornberg. Die Schüler vom Gätzi- und Warmesberg werden mit einem Bus nach Altstätten gebracht. Für den Schulbesuch ist aber nicht die Zugehörigkeit zu einer Schulgemeinde maßgebend, sondern die Wegdistanz. So besuchen z. B. die Kinder von Burst-Banriet die Schule in Krießern, obwohl ihre Väter die Steuern der Schulgemeinde Altstätten zahlen. Diese muß dafür der Schulgemeinde Krießern ein Schulgeld entrichten. 
Die Schulgemeinde ist verpflichtet, den unentgeltlichen Unterricht gemäß den kantonalen Gesetzen zu gewährleisten (8 Jahre Primarschule oder 6 Jahre Primar- und 3 Jahre Sekundarschule). Falls sie keine eigene Sekundarschule unterhält, muß sie durch Verträge mit einer Sekundarschule den Unterricht ermöglichen. Die katholische Sekundarschule in Altstätten wird darum von Schülern von Oberriet bis Rebstein besucht. Der Kindergarten wird in Altstätten durch einen privaten Verein organisiert, an den die Schul- und die Politische Gemeinde Beiträge zahlen.

Die Ausgaben der Schulgemeinde müssen durch die Schulsteuer, die Fondserträge und die kantonalen Subventionen gedeckt werden. Die Versammlung der Schulbürger beschließt jährlich über das Budget und den nötigen Steuerfuß. An die Lehrergehälter leistet der Kanton einen Beitrag, der vom Steuerfuß und Steuerertrag der Gemeinde abhängig ist. Die katholische Schulgemeinde Altstätten erhält 25\% der Primar- und $41 \%$ der Sekundarlehrergehälter Die Wahl der Lehrer ist durch das Erziehungsgesetz der Schulbürgerversammlung aufgetragen, die katholischen Schulgenossen von Altstätten haben das Wahlrecht aber an den Schulrat abgetreten.

\section{Die katholische Kirchgemeinde Altstätten}

Die älteste Kirche des Tales scheint in Marbach gestanden zu sein, wo neueste Ausgrabungen vorromanische Grundmauern zu Tage brachten. Altstätten, dessen Name 853 erstmals urkundlich erwähnt wird, besaß nur eine Filialkirche, die 1275 bezeugt ist. Erst um 1390 wurde Altstätten kirchlich von Marbach losgelöst. In einer Urkunde von 1421 wird Lüchingen politisch zu Altstätten, kirchlich aber zu Marbach geschlagen. 1528 trat die Gemeinde zum neuen Glauben über, 1531 setzten die regierenden Orte der Vogtei Rheintal aber die konfessionelle Parität durch, so daß beide Konfessionen nebeneinander bestanden. 1794 bis 1798 wurde eine neue Kirche gebaut, die bis 1906 paritätisch für die Gottesdienste benutzt wurde. Damals errichteten die Protestanten eine eigene Kirche.

Vor der Französischen Revolution bestand die Kirchenverwaltung aus den katholischen Mitgliedern des Kleinen Stadtrates; heute wird sie durch den Verwaltungsrat der Kirchgemeinde besorgt. Der Kirchgenossenversammlung stehen die folgenden Rechte zu: Wahl der Kirchenverwaltung, Beschlußfassung über Rechnung, Steueransatz, Bauten und Liegenschaften. Der Verwaltungsrat führt die Geschäfte der Kirchgemeinde, verwaltet das Vermögen und wählt die kirchlichen Angestellten (Sigrist usw.). Die katholische Kirchgemeinde Altstätten umfaßt die beiden Pfarreien Altstätten und Hinterforst, die je eine Kirche besitzen. Daneben sind noch die Klosterkirche im Kirlen bei Altstätten und die Kapellen auf dem Ruppen und dem Forst zu erwähnen. In Altstätten bestehen je eine Pfarr-, Kaplanei- und Vikariatspfrund, in Hinterforst eine Pfarrpfrund. Das Pfrundwahlrecht hat die Kirchgemeinde aber nur noch für die Pfarrpfrund in Altstätten, die übrigen Geistlichen bestimmt der Bischof von St. Gallen.

\section{Literaturverzeichnis}

\section{Gesetze:}

Verfassung des Kantons St. Gallen vom 16. November 1890 .

Gesetz über die Besorgung der Angelegenheiten des katholischen und des evangelischen Konfessionsteiles vom 25. Juni 1923.

Grundgesetz der Evangelischen Kirche des Kantons St. Gallen vom 9. Januar 1922.

Kirchenordnung der Evangelischen Kirche des Kantons St. Gallen vom 25. Juni 1923.

Organisation des katholischen Konfessionsteiles des

Kantons St. Gallen vom 3. Oktober 1939.

Gesetz über die Organisation und Verwaltung der Gemeinden und Bezirke und das Verfahren vor den Verwaltungsbehörden (Organisationsgesetz) vom 29. Dezember 1947.

Verordnung über die Bewirtschaftung und Nutzung der Ortsgemeindegüter vom 24. Dezember 1948.

Erziehungsgesetz vom 7. April 1952.

Ortsgemeinde Altstätten: Vereinbarung und Reglement über die Gemeindegüterverwaltung vom 14. Mai 1950.

\section{Úbrige Literatur}

Bader, K. S.: Studien zur Rechtsgeschichte des mittelalterlichen Dorfes, zweiter Teil: Dorfgenossenschaft und Dorfgemeinde, Köln 1962.

Kaplan Büchel: Das katholische Schulwesen in Altstätten vom Jahre 1800 bis zur Gegenwart, Altstätten 1925.

Eidgenössisches Statistisches Amt: Eidgenössische Volkszählung 1960, Band 14.

Schweizerisches Ortschaftenverzeichnis 1960, Bd. III.

Gächter, E.: Untersuchungen zur agrargeographischen Struktur des St. Galler Oberrheintales, Manuskript Geographisches Institut Universität Zürich 1966.

Rohner, J.: Altstätten, aus der Geschichte des rheintalischen Marktstädtchens, Altstätten 1937.

Rohner, O.: Die Rheintalischen Ortsgemeinden, Jahrbuch Unser Rheintal 1967.

Rohner, W., Müller, H.: Altstätten im Wandel der Zeit, Altstätten 1953.

Schöbi, J.: Die St. Gallischen Ortsgemeinden, geschichtlich-dogmatische Darstellung, Altstätten 1934. 


\section{Summary}

This article deals with the concept and the mode of existence of the community (Gemeinde) in Switzerland. Following a brief discussion of the origin of the different communities and their present legal groundwork, the significance of individual communities is illustrated by an example - Altstätten in the Rhine Valley (the canton of St. Gall).

In addition to the Politische Gemeinde, there exist the Ortsgemeinde which consists of niniteen subdivisions called Rhoden, ten denominational Schulgemeinden and two Roman Catholic and one Protestant Kirchgemeinde.

The Politische Gemeinde (political community) performs all the public tasks of the community such as the police, the fire brigade, public utilities, land registry, etc.
The Ortsgemeinde (organisation of the citizens c Altstätten) has charge of its own extensive lande property, and reserves the right to decide upon th matters concerned with naturalization and citizer ship.

The Schulgemeinde (school community) is respor sible for organizing the eight- to nine-year educatio for children with no charge.

The Kirchgemeinden (parishes) takes care of churc affairs.

Every resident of Altstätten is a member of the $P C$ litische Gemeinde and a Schulgemeinde, and, if $\mathrm{h}$ belongs to one of the two confessions, also of Kirchgemeinde. Only if he is the citizen of Altstä1 ten, he has a membership in the Ortsgemeinde a well. 